\title{
The Optical Measurement and Quantitative Analysis of Algesia in Spodoptera litura Larva
}

\author{
Ying-Yun Chen ${ }^{1}{ }^{*}$, Rong-Seng Chang ${ }^{1}$, Mi-Yin Tsai ${ }^{1}$, and Der-Chin Chen $^{2}$ \\ ${ }^{1}$ Department of Optics and Photonics, National Central University, Taoyuan 32001, Taiwan \\ ${ }^{2}$ Department of Electrical Engineering, Feng Chia University, Taichung 40724, Taiwan
}

(Received December 4, 2014 : revised February 16, 2015 : accepted March 9, 2015)

\begin{abstract}
Muscle vibration measurement has long been an unique scientific study, in general, and the direct reaction of animals to feel pain (algesia), either from vascular or muscle contraction, is a complex perceptual experience. Thus this paper proposes a way to measure animal algesia quantitatively, by measuring the changes in muscle vibration due to a pinprick on the surface of the skin of a Spodoptera litura larva. Using the laser optical triangulation measurement principle, along with a CMOS image sensor, linear laser, software analysis, and other tools, we quantify the subtle object point displacement, with a precision of up to $10 \mu \mathrm{m}$, for our chosen Spodoptera litura larva animal model, in which it is not easy to identify the tiny changes in muscle contraction dynamics with the naked eye. We inject different concentrations of formalin reagent (empty needle, $12 \%$ formalin, and $37 \%$ formalin) to obtain a variety of different muscle vibration frequencies as the experimental results. Because of the high concentrations of reagent applied, we see a high frequency shift of muscle vibration, which can be presented as pain indices, so that the algesia can be quantified.
\end{abstract}

Keywords : Algesia, Laser optical triangulation, Formalin

OCIS codes : (140.3460) Lasers; (100.2000) Digital image processing; (170.1420) Biology

\section{INTRODUCTION}

For an animal, algesia is a complex perceptual experience. Putting aside the cause of the algesia and the pathway of message transduction, mostly it is expressed by means of body twitching and muscle or vascular contraction, when an animal is subjected to certain external stimuli, and it cannot be expressed in words or actions of such unpleasant degree. Most experimental research on animal algesia is the observation of biological responses to pain, such as tail swinging, screaming, and twitching rates of limbs, but these behaviors are unstable and less objective measurements [1-11].

In previous studies, there have been many algesia experiments using acidic reagents to cause pain, and formalin has been found to have the strongest effect for causing feelings of pain in animals. For example, Rosland and Candelletti injected formalin into mice $[12,13]$; Shima adapted it to cats [14]; Alreja chose primates [15]; Carli used rabbits [16]; Takahashi used guinea pigs in experiments [17]; Kanui used crocodiles [18]; Hughes focused on domestic fowl [19]; and Pelissier selected Octodon degus [20]. All of them chose formalin as a pain reagent for different kinds of animals, and have provided us sufficient research data. Thus, in this study we, too, choose formalin.

Most previous studies used low concentrations of formalin, ranging from 10 to $15 \%$ [12-20]. Nonetheless, Oyadeyi considered that using lower formalin concentrations did not produce easily detectable responses [21], so he chose $37 \%$ formalin in his experiment. In our study we used two concentrations: We chose $12 \%$, the average from 10 to $15 \%$ in previous papers, as our low concentration, and $37 \%$ as our high concentration, according to Oyadeyi's research. The use of an optical measurement system in this paper proposes a way to measure animal algesia, using a CMOS image sensor, linear red laser optics, and the software analysis of the composition of the laser triangulation, as the measurement tools. We inject several different concentrations of formalin into our chosen animal model, Spodoptera litura larva; we measure the changes in patterns of muscle vibration frequency in the larvae due to the stimulation of the formalin reagent,

\footnotetext{
*Corresponding author: yychen2014@gmail.com

Color versions of one or more of the figures in this paper are available online.
} 
with respect to the observation of the reaction to a parasite stimulus for a positive correlation, and the comparison of different concentrations of formalin injection; and these signals are collected for further processing and to quantify the degree of pain.

\section{METHODS AND MATERIALS}

\subsection{Methods}

We use the laser optical triangulation technology, which is a noninvasive, noncontact measurement device for muscle vibration. The vibration information of a muscle can be obtained with this measurement system. An algorithm to evaluate the muscle vibration activities from the center of the laser spot intensity on a certain wrist point has been conducted by Fast Fourier Transform (FFT). The amplitude and frequency of muscle vibration can be read via this measurement system. The basic principle of the optical noncontact pain point measurement device is to project a spot of light onto a muscle surface, and then define the amplitude and frequency of muscle vibration by processing the scattered light. Our system adopts a simple structure based on laser optical triangulation, the geometrical layout is shown in Fig. 1, in which we define the following quantities.

$X$ is the distance between the laser collimator lens and the tested object; $\Delta \mathrm{X}$ is the amplitude of the wrist pulse vibration;

$L$ is the distance between the laser and the CMOS image sensor; $d$ is the distance between the two bright spots projected onto the CMOS image sensor; $f$ is the focal length of the lens; $Z$ is the distance between the center point $C$ of the lens in the CMOS image sensor and the baseline;

$\alpha$ is the angle between the tested spot $A$ and the central axis of the lens in the CMOS image sensor;

$\delta X^{\prime}$ is the distance of the central axis between the tested spot $A$ and the central axis of the lens in the CMOS image sensor;

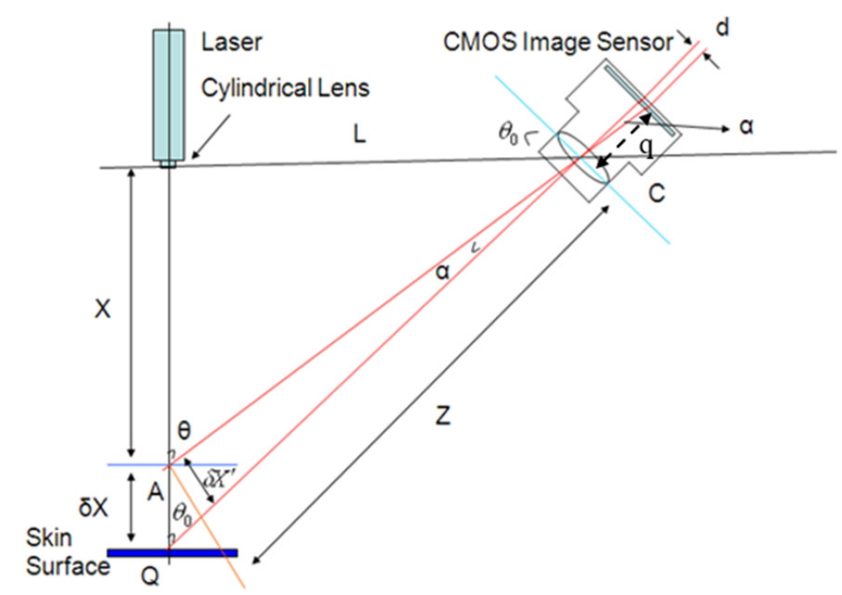

FIG. 1. Geometrical layout of the laser triangulation. $\theta_{0}$ is the angle between the laser at the baseline and the CMOS image sensor; and

$\mathrm{Q}$ is the intersection of the optical axis of the camera lens and the laser light axis.From Fig. 1 we learn that in $\triangle \mathrm{ABQ}, \delta X$ is very small (tiny vibration), so that $\theta_{0}$ can be taken as approximately equal to $\theta$ to obtain the relationship between $\delta X^{\prime}$ and $\delta X$.

$$
\frac{\delta X^{\prime}}{\delta X}=\sin \theta_{0}=\sin \theta
$$

Using similar triangles to replace equation (1),

$$
\frac{\delta X^{\prime}}{\delta X}=\frac{L}{Z}
$$

In $\triangle \mathrm{ABC}$,

$$
\alpha=\frac{\delta X^{\prime}}{Z}
$$

The geometry after the lens is

$$
\alpha=\frac{d}{f}
$$

From equations (3) and (4) we see that

$$
\delta X^{\prime}=\frac{Z d}{f}
$$

From equation (2) we see that $\delta X^{\prime}=\frac{\delta X}{Z} L$, and input (5) to obtain

$$
\delta X=\frac{Z^{2} d}{L f}
$$

From equation (6) we see that the shorter the tested distance, the higher the resolution, and $\frac{Z^{2}}{L f}$ is a constant, so $\delta X$ is directly proportional to $d$, indicating a linear relationship between the two. When the pulse vibrates at $\delta X$, the laser spot moves a proportional distance $d$ on the CMOS image sensor.

We can analyze the image on the CMOS sensor to obtain the muscle vibration amplitude. By setting a threshold level, any pixel with an intensity below that level will be neglected. The threshold gray level was set to $80 \%$ at the highest level in these experiments. The centroid method has been widely used to locate a light spot on image features of various types, to subpixel accuracy. By introducing subpixel processing, the resolution of this system can be increased. 
The intensity of the $i$ th pixel in a row of pixels on the sensor is $g_{i}(x)$. The center of the spot $\bar{X}_{c}$ is calculated as the centroid of $g_{i}$ between pixels $m$ and $n$. The centroid $\bar{X}_{c}$ of the laser spot, in one dimension, can be derived using

$$
\bar{X}_{c}=\left(\sum_{m}^{n} x_{i} g_{i}\right) /\left(\sum_{m}^{n} g_{i}\right)
$$

After using FFT (Fast Fourier Transform) on the waveform of the algesia point variation, we obtain the spectrum in frequency space, from which we can extract the peak frequency, i.e. the main vibration frequency due to the stimulation $[22,23]$.

\subsection{Materials and Sample Preparation}

We selected as our biological model the cutworm Spodoptera litura. The Spodoptera burglar is an omnivorous insect, thus it can be found very easily throughout the year. For the experiment we chose specimens at the adaptable age of 5 years, with a body length of $33-50 \mathrm{~mm}$. A suitable amount of synthetic rubber resin was used to fix the abdomen of the Spodoptera litura larva on a clean acrylic seat for testing. This synthetic rubber resin is a gelatinous, highly viscous, non-irritating sticky reagent used to confine the larva at a certain position while not completely hindering its movement, to facilitate dynamic measurements.

\subsection{Experimental Methods and Procedures}

In this study we chose a red diode laser modulewith a wavelength of 635-680 $\mathrm{nm}$ and an output power of $3 \mathrm{~mW}$; in the part of the CMOS image sensor, its image resolution is $1280 \times 720$ pixels and the shooting speed is 30 frames/second.

The experimental framework is set up with a fixed optical laser line by the cylindrical lens of the laser light source, and the object to be tested is placed in the line of the laser. Therefore, during measurement the subject must stay within the scope of the laser line, which is the only region that can be measured; even though the synthetic rubber resin was used to fix the abdomen of the larva, its head and tail struggled severely and nonetheless occasionally deviated from the laser line. Only the larval body section in the middle part of the abdomen (the 7th somite of the body, for larvae of 13 somites) struggled relatively moderately, such that it would not deviate from the optical line. Therefore, the injection site was the abdominal blood vessel of the larva at the center of the back, as shown in Fig. 3. Chemicals were injected into the five-year-old larva's abdomen, near the back of the blood vessel, a distance of about $1 \mathrm{~mm}$, subcutaneously approximately $1 \mathrm{~mm}$. The back of the Spodoptera litura larva's body wall is thin, the space between the membrane has no the epidermis, and the outer epidermis, which is the softest part of the body wall, is suitable for a glass capillary needle. To avoid significant blood loss, the larva is pricked about $1 \mathrm{~mm}$ to

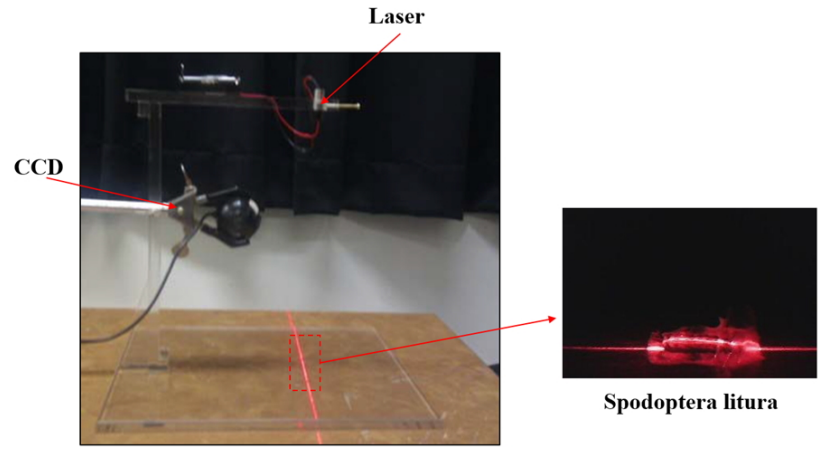

FIG. 2. A laser line is projected onto vibrating muscle of a Spodoptera litura larva.

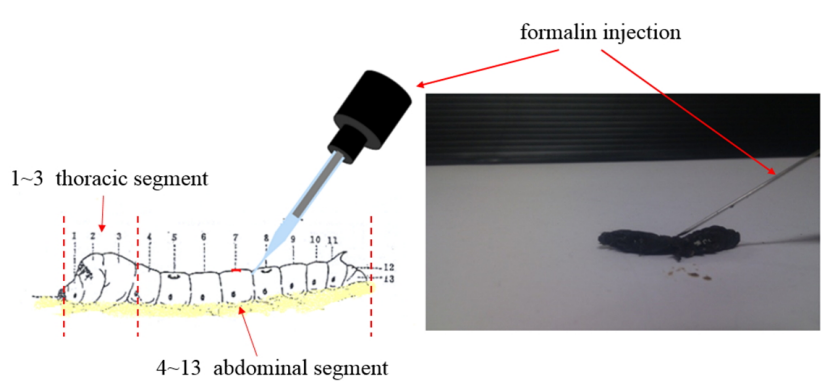

FIG. 3. Schematic diagram of the injection site.

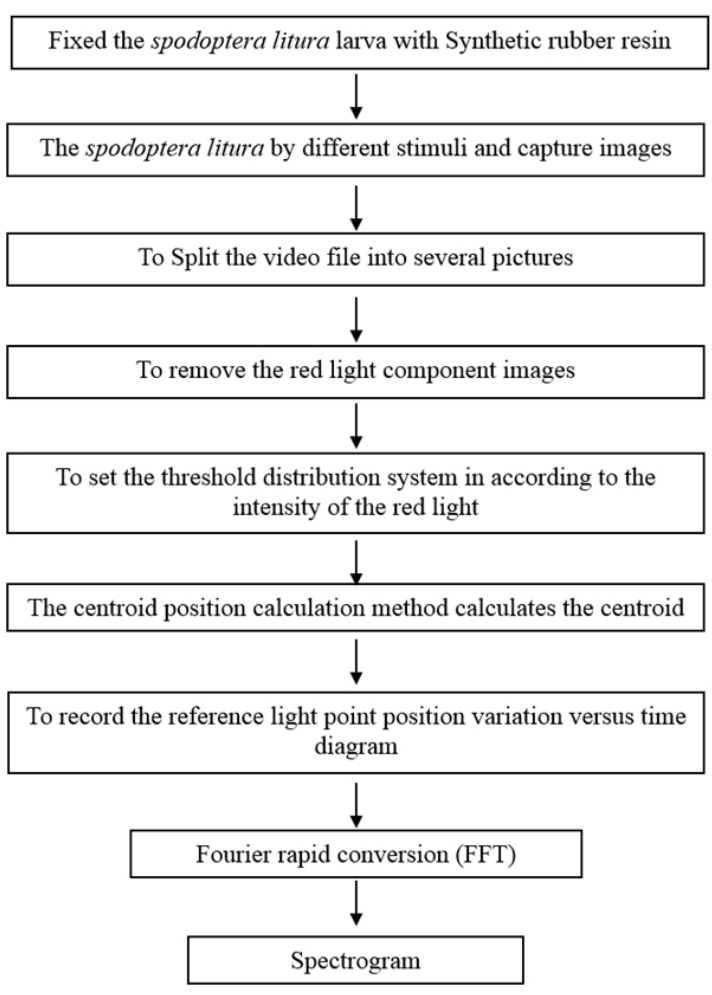

FIG. 4. Flowchart for the experiments.

the left of the blood vessel.

The study was performed by injecting of two different 
concentrations of reagents into 30 different five-year-old Spodoptera litura larva, followed by recording their muscle vibrations for 20 seconds each, with $12 \%$ and $37 \%$ formalin as the two reagents to stimulate the muscle contractions, in addition to an empty injection for a control group, for a total of three experiments. After the larva is injected from above, the injection site on the forms a linear laser line on the skin surface, as shown in Fig. 2, the laser line vibration is recorded by the CMOS camera, the program calculates the optical centroid, and the software for the Fourier spectral analysis obtains the spectrum for the muscle vibration at the injection site. A flowchart for this process is shown in Fig. 4.

\subsection{System Calibration and Error Analysis}

To increase the reliability of the experimental data, before the measurement of the muscle vibration we put a horn on the acrylic plate and projected a laser line on the surface of the horn to measure the vibration frequency of the horn, generated by a signal generator. We then compared our measurement results with the original signal generator output to determine the errors of our measurements. The signal generator output frequency is accurate to one decimal point (but the frequency from the frequency signal generator cannot be a fixed value, as it drifts over time), and we adjusted the output frequency from 0.60 to $2.00 \mathrm{~Hz}$ in steps of $0.10 \mathrm{~Hz}$. Figure 5 is a schematic diagram of the frequency calibration experiment, while Fig. 6 shows the actual setup of the calibration system. Based on our need to measure

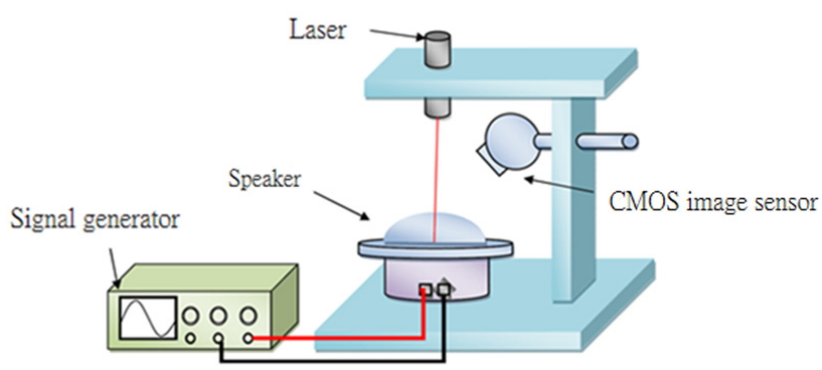

FIG. 5. Schematic diagram of the frequency calibration experiment.

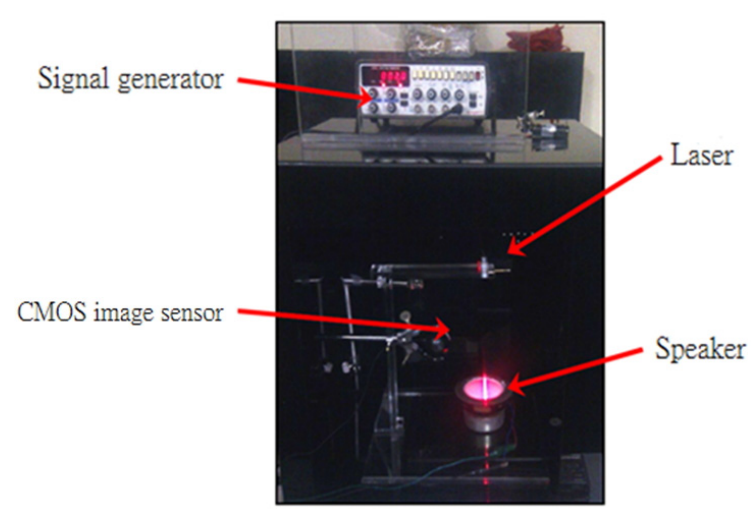

FIG. 6. Actual setup of the calibration system. from 0.60 to $2.0 \mathrm{~Hz}$, the calibration experiments show that the error are less than $3 \%$, as presented in Table 1 .

\section{EXPERIMENTAL RESULTS}

Figures 7 and 8 present for each repeated experiment the muscle vibration amplitudes versus frequencies and the main frequencies for the Spodoptera litura larva. The average over 30 trials for the main (peak) frequency value is 0.99 $\mathrm{Hz}$ for the algesia sensation after an empty injection, 1.36 $\mathrm{Hz}$ after $12 \%$ formalin reagent injection, and $1.95 \mathrm{~Hz}$ after

TABLE 1. Calibration of the detection system for frequencies 0.60 to $2.00 \mathrm{~Hz}$

\begin{tabular}{c|c|c}
\hline $\begin{array}{c}\text { Input Frequency } \\
(\mathrm{Hz})\end{array}$ & $\begin{array}{c}\text { Measured } \\
\text { Frequency }(\mathrm{Hz})\end{array}$ & $\begin{array}{c}\text { Errors } \\
(\%)\end{array}$ \\
\hline 0.60 & 0.59 & $1.67 \%$ \\
\hline 0.70 & 0.72 & $2.85 \%$ \\
\hline 0.80 & 0.82 & $2.50 \%$ \\
\hline 0.90 & 0.88 & $2.22 \%$ \\
\hline 1.00 & 1.03 & $3.00 \%$ \\
\hline 1.10 & 1.11 & $0.91 \%$ \\
\hline 1.20 & 1.23 & $2.50 \%$ \\
\hline 1.30 & 1.29 & $0.77 \%$ \\
\hline 1.40 & 1.41 & $0.71 \%$ \\
\hline 1.50 & 1.52 & $1.33 \%$ \\
\hline 1.60 & 1.58 & $1.25 \%$ \\
\hline 1.70 & 1.74 & $2.35 \%$ \\
\hline 1.80 & 1.82 & $1.11 \%$ \\
\hline 1.90 & 1.87 & $1.58 \%$ \\
\hline 2.00 & 2.02 & $1.00 \%$ \\
\hline Average & & $1.72 \%$ \\
\hline
\end{tabular}

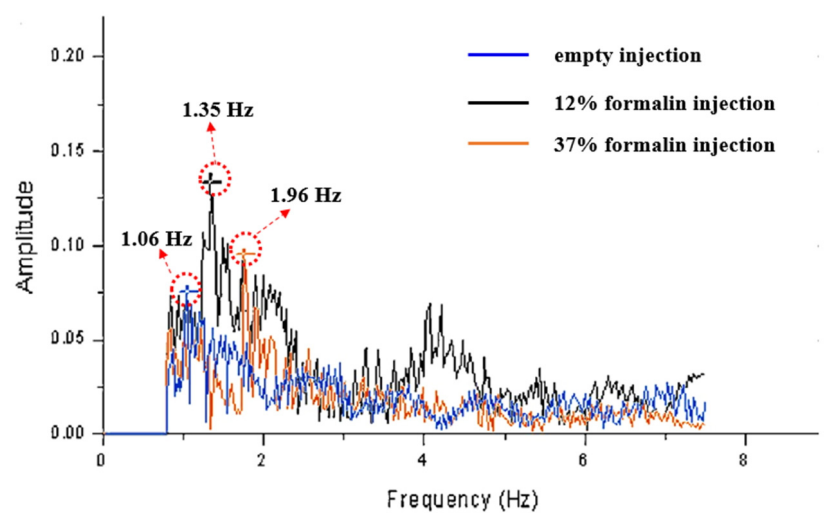

FIG. 7. Frequency graph for five-year-old Spodoptera litura larva. Solid lines in different colors show the frequencies resulting from empty, $12 \%$ formalin, and $37 \%$ formalin injections. In each frequency analysis the highest amplitude is selected as the main frequency, which is marked by a red dotted circle, and represents the reaction of Spodoptera litura larva. 


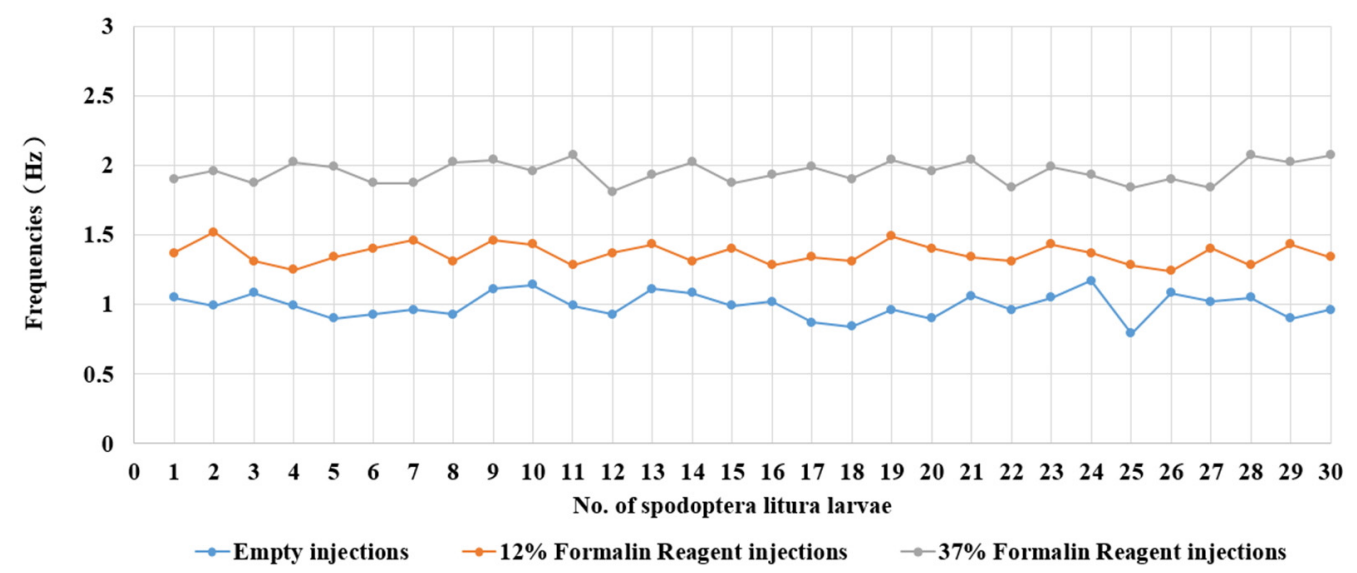

FIG. 8. Peak muscle vibration frequencies of Spodoptera litura larva. The experiment was repeated 30 times for each formalin reagent level (empty injection, 12\% formalin, and 37\% formalin). Results are shown as solid lines in different colors. The average of each main (peak) frequency is $0.99 \mathrm{~Hz}, 1.36 \mathrm{~Hz}$, and $1.95 \mathrm{~Hz}$ respectively.

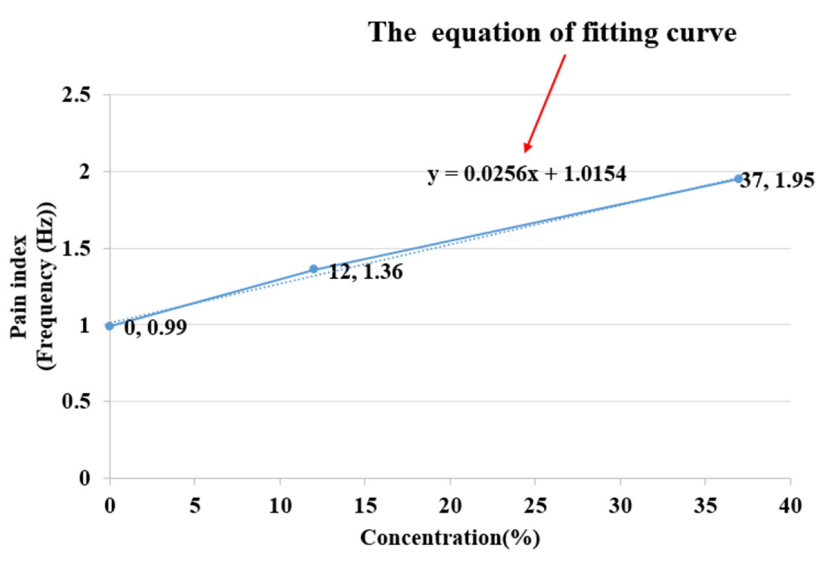

FIG. 9. Correlation between formalin concentration and pain index (peak frequency). The solid line illustrates the relationship between concentration and pain index, which is the main (peak) frequency of muscle contraction. The dotted line is the fitted curve, a line, and the equation of the fitted curve is displayed. We can calculate the pain index for a different concentration of formalin injected into Spodoptera litura by using this fitted equation.

$37 \%$ formalin reagent injection. Compared to the empty injection, there is a positive shift in frequency of $0.37 \mathrm{~Hz}$ for the $12 \%$ formalin reagent injection and $0.96 \mathrm{~Hz}$ for the $37 \%$ formalin reagent injection.

Here we use the statistical $t$-test to assess the difference between the empty injection and formalin injections. To test the significant of the difference, we set up the hypothesis 0 (H0) that the muscle vibration frequency following an empty injection is the same as that following a $12 \%$ or $37 \%$ formalin injection. Our hypothesis $1(\mathrm{H} 1)$ is that the muscle vibration frequency for a $12 \%$ or $37 \%$ formalin reagent injection is higher than that for an empty injection. We set our significance level to be 0.01 (alpha $=1 \%$ ). If the $p$-value is less than alpha, we reject hypothesis 0 and accept hypothesis
1. Since the $p$-value for comparing empty and $12 \%$ formalin injections is $7 \times 10^{-25}$, and that for comparing empty and $37 \%$ formalin injections is $2.9 \times 10^{-46}$, both far less than our significance level of 0.01 , we reject $\mathrm{H} 0$ and accept $\mathrm{H} 1$ : The muscle vibration frequency for formalin injections is significantly higher than that for an empty injection.

The comparison of different concentrations of reagents displays a similarly high muscle vibration of the exponential increase on the algesia in Spodoptera litura larva.

Here we conclude that algesia causes muscle contraction; the more pain, the more frequently muscle contractions occur. The results are summarized in Figs. 7 through 9. According to our measurements, the main (peak) frequency and formalin concentration (pain feeling) have a positive correlation.

\section{DISCUSSION}

In Figure 7, we find that no regular pattern in amplitude is observed in our experiments. When Spodoptera litura feels pain, it contracts its muscles to ease the feeling; however, when the feeling is too strong, partial muscle contraction is not enough and spodoptera litura needs to twitch its whole body to decrease the pain.

The energy causing its muscle contraction, in this extreme case, becomes body twitch, so the amplitude of the main (peak) frequency diminishes. In Fig. 7 the amplitude of the peak frequency for $37 \%$ formalin injection is less than that for $12 \%$. Meanwhile, our measurement only focuses on partial muscle contraction, not the whole body. Therefore, in this study we choose the main (peak) frequency, not the amplitude, to be the pain index.

Meanwhile, since the muscle contraction is not a pure sine curve but a complicated distortion, the Fast Fourier Transform generates other peaks. In this study we focus on the main frequency because it has a more significant relation to pain, and we leave the study of other peaks for the future. 
This study selects the muscle vibration frequency of Spodoptera litura to be the pain index. We plot the correlation between formalin concentration and pain index (peak frequency) and develop an equation by fitting a curve, as shown in Fig. 9. The higher the formalin concentration, the higher is the main (peak) frequency, meaning a higher pain index. We can calculate the pain index for a different concentration of formalin injected into Spodoptera litura by using this fitted equation.

\section{CONCLUSION}

In this study, a CMOS image sensor and the linear laser optical triangulation method were used as measurement tools, with software for image processing and data analysis. We obtained a pain index quantity. We chose Spodoptera litura larva as the experimental animal model for the injection of different concentrations of chemicals (12\% and $37 \%$ formalin) to stimulate muscle vibration. The results show that treatment with different concentrations of reagents yields different muscle vibration spectra, and that there is an equation for the relation between formalin concentration (feeling of pain) and pain index (peak frequency) to quantify the pain feeling. This system will be used to quantify algesia in other creatures, and yet the noncontact optical measurement does not damage the surface, thus reducing unnecessary interference in the measurement, and is superior to the general mechanical-probe measurement method, with high accuracy and repeatability as well.

\section{ACKNOWLEDGMENT}

This study was supported by a grant from The Aim For The Top University Project of Taiwan (no.103G912-1)

\section{REFERENCES}

1. E. H. Hofmeister, J. King, M. R. Read, and S. C. Budsberg, "Sample size and statistical power in the small-animal analgesia literature," Journal of Small Animal Practice 48, 76-79 (2007).

2. A. Tjølsen and K. Hole, "Animal models of analgesia," The Pharmacology of Pain 130, 1-20 (1997).

3. L. F. Vendruscolo and R. N. Takahashi, "Synergistic interaction between mazindol, an anorectic drug, and swim-stress on analgesic responses in the formalin test in mice," Neurosci. Lett. 355, 13-16 (2004).

4. S. Louisa, "Considerations for prospective studies in animal analgesia," Veterinary Anaesthesia and Analgesia 37, 303-305 (2010).

5. I. Hiroki, I. L. Yasutomo, and S. Emiko, "Stress-induced hyperalgesia: Animal models and putative mechanisms," Frontiers in Bioscience 11, 2179-2192 (2006).

6. J. S. Mogil, "Animal models of pain: progress and challenges," Nature Reviews Neuroscience 10, 283-294 (2009).
7. F. V. Abbott, K. B. J. Franklin, R. J. Ludwick, and R. Melzack, "Apparent lack of tolerance in the formalin test suggests different mechanisms for morphine analgesia in different types of pain," Pharmacology Biochemistry and Behavior 15, 637-640 (1981).

8. M. S. Fanselow, "Shock-induced analgesia on the formalin test: Effects of shock severity, naloxone, hypophysectomy, and associative variables," Behavioral Neuroscience 98, 79-95 (1984).

9. A. E. Desjardins, B. H. W. Hendriks, M. Voort, R. Nachabé, W. Bierhoff, G. Braun, D. Babic, J. P. Rathmell, S. Holmin, M. Söderman, and B. Holmström, "Epidural needle with embedded optical fibers for spectroscopic differentiation of tissue: Ex vivo feasibility study," Biomedical Optics Express 2, 1452-1461 (2011).

10. K. Hargreaves, R. Dubner, F. Brown, C. Flores, and J. Joris, "A new and sensitive method for measuring thermal nociception in cutaneous hyperalgesia," Pain 32, 77-88 (1988).

11. S. T. Kooa, Y. I. Parka, K. S. Lima, K. Chunga, and J. M. Chunga, "Acupuncture analgesia in a new rat model of ankle sprain pain," Pain 99, 423-431 (2002).

12. J. H. Rosland, "The formalin test in mice: The influence of ambient temperature," Pain 45, 211-216 (1991).

13. S. Candelletti and S. Ferri, "Antinociceptive profile of intracerebrovascular salmon calcitonin and calcitonin-gene related peptide in the mouse formalin test," Neuropeptides 17, 93-98 (1990).

14. K. Shima, H. Nakahama, M. Yamanoto, K. Aya, and M. Inase, "Effects of morphine on two types of nucleus raphe dorsalis neurons in awake cats," Pain 29, 375-386 (1987).

15. M. Alreja, P. Mutalik, U. Nayar, and S. K. Manchanda, "The formalin test: A tonic pain model in the primate." Pain 20, 97-105 (1984).

16. G. Carli, F. Farabollini, and G. Fontani, "Effects of pain, morphine and naloxone on the duration of animal hypnosis," Behav. Brain Res. 2, 373-385 (1981).

17. H. Takahashi, K. Shibata, T. Ohkubo, and S. Naruse, "Formalin induced minor tremor response as an indicator of pain," Nippon Yakurigaku Zasshi 84, 353-362 (1984).

18. T. I. Kanui, K. Hole, and J. O. Miaron, "Nociception in crocodiles: Capsaicin instillation in formalin and hot plate tests," Zool. Sci. 7, 537-540 (1990).

19. R. A. Hughes and J. Sulka, "Morphine hyperalgesic effects on the formalin test in domestic fowl," Pharmacol. Biochem. Behav. 38, 247-251 (1991).

20. T. Pelissier, H. Saavedra, D. Bustamante, and C. Panicle, "Further studies on the understanding of Octodon degus natural resistance to morphine; A comparative study with the Wistar rat," Comp. Biochem. Physiol. 192, 319-322 (1989).

21. A. S. Oyadeyi, F. O. Ajao, A. O. Afolabi, U. S. Udoh, and O. M. Azeez, "The formalin test in African toad (Bufo regularis) - A novel pain model in amphibians," AmericanEurasian Journal of Scientific Research 2, 24-28 (2007).

22. R. S. Chang, J. H. Chiu, F. P. Chen, J. C. Chen, and J. L. Yang, "A Parkinson's disease measurement system using laser lines and a CMOS image sensor," Sensors 11, 1462-1475 (2011).

23. J. H. Wu, R. S. Chang, and J. A. Jiang, "A novel pulse measurement system by using laser triangulation and a CMOS image sensor," Sensors 7, 3366-3385 (2007). 